Rechtsmedizin 2020 · 30:144-152 https://doi.org/10.1007/s00194-020-00372-6 Online publiziert: 23. März 2020

(C) Der/die Autor(en) 2020

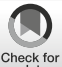

C. Dieterich ${ }^{1}$ - E. Herrmann ${ }^{2} \cdot$ M. Parzeller ${ }^{1}$

'Institut für Rechtsmedizin, Universitätsklinikum, Goethe-Universität Frankfurt a. M., Frankfurt a. M., Deutschland

${ }^{2}$ Institut für Biostatistik und Mathematische Modellierung, Zentrum der Gesundheitswissenschaften, Klinikum und Fachbereich Medizin, Goethe-Universität Frankfurt a. M., Frankfurt a. M., Deutschland

\title{
Tod bei der Arbeit - eine Analyse tödlicher Arbeitsunfälle von 2005 bis 2016 im Obduktionsgut des Instituts für Rechtsmedizin in Frankfurt am Main
}

Auf dem 20. Weltkongress für Sicherheit und Gesundheit bei der Arbeit wurde 2014 das Konzept einer Welt ohne tödliche Arbeitsunfälle diskutiert. Dieses Konzept ist unter dem Begriff "vision zero" bekannt. Laut der Internationalen Arbeitsorganisation, einem der Hauptveranstalter des Kongresses, ereignen sich weltweit mehr als 313 Mio. Arbeitsunfälle/Jahr. Der Anteil der davon tödlich verlaufenden Arbeitsunfälle wird mit über $\mathbf{3 5 0 . 0 0 0}$ Fällen angegeben [14].

In Deutschland ist die Anzahl tödlicher Arbeitsunfälle nach einer Erhebung der Bundesanstalt für Arbeitsschutz und Arbeitsmedizin in dem Zeitraum von 1991 bis 2016 von 1496 auf 557 Unfälle zurückgegangen. Seit dem Jahr 2009 stagniert der zuvor kontinuierliche Rückgang tödlicher Arbeitsunfälle. Zuletzt hat die Anzahl tödlicher Arbeitsunfälle für das Jahr 2017 entgegen dem Trend auf 564 Fälle zugenommen [31]. Das genaue Wissen um die Umstände, Abläufe und Folgen eines jeden tödlichen Arbeitsunfalls ist Voraussetzung für die Identifizierung von Unfallschwerpunkten und ermöglicht eine effektive Präventionsarbeit im Sinne der „vision zero".

Nicht jedes tödliche Ereignis, das während der Arbeitszeit eintritt, ist ein töd- licher Arbeitsunfall. Arbeitsunfälle sind gemäß $₫ 8$ Abs. 1 S. 2 SGB VII (Sozialgesetzbuch VII) Unfälle von Versicherten, die durch ein zeitlich begrenztes, von außen auf den Körper einwirkendes Ereignis zu einem Gesundheitsschaden oder zum Tode führen. Tödliche Arbeitsunfälle im Sinne des SGB VII setzen gemäß $\$ 8$ Abs. 1 S. 1 SGB VII eine den Versichertenschutz begründende Tätigkeit, die sog. versicherte Tätigkeit, nach den $\$ \$ 2,3$ oder 6 SGB VII sowie nach $₫ 8$ Abs. 2 SGB VII (z. B. Wegeunfall) voraus. Voraussetzung für die Anerkennung eines Arbeitsunfalls ist in der Regel ein innerer Zusammenhang zwischen der versicherten und der unfallbringenden Tätigkeit. Nach der ständigen Rechtsprechung des Bundessozialgerichts (BSG) ist für die Unfallkausalität und die haftungsbegründende Kausalität erforderlich, dass die Verrichtung der versicherten Tätigkeit den Tod bzw. den Gesundheitserstschaden beim Versicherten „objektiv und rechtlich wesentlich“ verursacht hat (BSG, Urt. v. 27.11.2018 - B 2 U 15/17 R mwN).

Die Ergebnisse einer gerichtsmedizinischen Sektion sind wertvolle Beweismittel für den Nachweis der oben genannten Kausalzusammenhänge von Tätigkeit, Unfallereignis und Todesfolge. Gelegenheitsursachen, natürliche Todesfälle, Suizide und Tötungsdelikte lassen sich meist erst durch eine gerichtliche Lei- cheneröffnung ausschließen. Die plötzlich eintretende Bewusstlosigkeit eines Dachdeckers mit nachfolgendem Sturz aus großer Höhe ist beispielsweise eine potenzielle Gelegenheitsursache. In diesem Fall lässt sich nur anhand von Sektionsergebnissen eruieren, ob das äußere Ereignis des Absturzes oder eine akute innere Erkrankung des Arbeiters die rechtlich wesentliche Todesursache ist. Ein spektakuläres Beispiel für eine vorsätzliche Tötung unter Vortäuschung eines Arbeitsunfalls geben Betz und Eisenmenger in ihrer rechtsmedizinischen Obduktionsstudie von 1992 [5].

\section{Material und Methode}

In der vorliegenden Studie wurden die tödlichen Arbeitsunfälle für einen Zwölfjahreszeitraum aus den Jahren 2005 bis 2016 untersucht, die sich im Einzugsbereich der Rechtsmedizin Frankfurt am Main (Raum Frankfurt, Offenbach, Darmstadt, Wiesbaden, Hanau, Südhessen) ereigneten und im Institut der Rechtsmedizin des Universitätsklinikums der Goethe-Universität Frankfurt am Main obduziert worden sind. Nicht berücksichtigt wurden Wegeunfälle mit tödlichem Ausgang. Hierbei handelt es sich um Unfälle auf dem Weg von oder zur Arbeitsstelle ( $\$ 8$ Abs. 2 SGB VII).

Zur Auswertung kamen die Sektionsprotokolle und die dem Institut für 
Tab. 1 Praktisches Beispiel für die Berechnung des International Severity Score (ISS) anhand der mittels Abbreviated Injury Scale (AIS) codierten

Schwere des Verletzungsbildes eines Unfallopfers

\begin{tabular}{|c|c|c|c|c|c|c|c|}
\hline $\begin{array}{l}\text { Verletzungen nach } \\
\text { AIS-Code }\end{array}$ & Körperregion I & Schweregrad I & Körperregion II & Schweregrad II & Körperregion IIII & Schweregrad III & ISS \\
\hline 140662.3 & \multirow[t]{11}{*}{ Bauch } & \multirow[t]{11}{*}{4} & \multirow[t]{11}{*}{ Brust } & \multirow[t]{11}{*}{3} & \multirow[t]{11}{*}{ Kopf, inkl. Gesicht } & \multirow[t]{11}{*}{3} & \multirow[t]{11}{*}{34} \\
\hline 310099.1 & & & & & & & \\
\hline 410604.2 & & & & & & & \\
\hline 442205.3 & & & & & & & \\
\hline 441430.3 & & & & & & & \\
\hline 441411.3 & & & & & & & \\
\hline 450203.3 & & & & & & & \\
\hline 541826.4 & & & & & & & \\
\hline 541610.2 & & & & & & & \\
\hline 650416.2 & & & & & & & \\
\hline 650499.2 & & & & & & & \\
\hline ISS = (AIS-Körperregio & I) $)^{2}$ (AIS-Körperres & n II) $)^{2}+($ AIS-Körp & egion $\| I)^{2}$ & & & & \\
\hline
\end{tabular}

Rechtsmedizin zur Verfügung gestellten polizeilichen Ermittlungsakten. Bei unvollständigen Angaben wurde bei den zuständigen Staatsanwaltschaften auf Basis eines spezifischen Datenschutzkonzepts um Akteneinsicht gebeten.

Es wurden Alter, Geschlecht, Nationalität, Wirtschaftszweig, Unfallzeitpunkt, Unfallmechanismus, Verletzungen und Todesursache der Unfallopfer untersucht.

Um das Körpergewicht zur Körpergröße in Relation zu setzen, wurde der Body-Mass-Index (BMI) berechnet. Der BMI ist der Quotient aus Gewicht und Körpergröße zum Quadrat $\left(\mathrm{kg} / \mathrm{m}^{2}\right)$. Die Einteilung der BMI-Werte erfolgte nach der Gewichtsklassifikation der World Health Organization in Normalgewicht (BMI 18,5-24,9 kg/m²), Präadipositas (BMI 25,0-29,9 kg/m²), Adipositas Grad I (BMI 30,0-34,9 kg/m²) und Adipositas Grad II (BMI 35,0-39,9 kg/m²) [32].

Die toxikologische Untersuchung der Unfallopfer wurde im Hinblick auf Blutalkoholkonzentration (BAK) und Drogenmissbrauch ausgewertet. Zur Bestimmung der BAK wurde das aus der Femoralvene entnommene Leichenblut gaschromatographisch untersucht.

Zur Kodierung der in den Obduktionsprotokollen dokumentierten Verletzungen wurde nachträglich im Rahmen dieser Studie die Abbreviated Injury Scale (AIS) angewandt [13]. Die AIS ermöglicht eine objektive Klassifizierung und Vergleichbarkeit von Verletzungen unterschiedlicher Körperregionen in Bezug auf ihre Letalität und wird in der Notfallmedizin zur Beurteilung polytraumatisierter Patienten eingesetzt. Um einen vergleichbaren Wert für die ganzheitliche Verletzungsschwere eines jeden Unfallopfers zu erhalten, wurde anschließend der International Severity Score (ISS) berechnet $[3,13]$. Ein praktisches Beispiel für die Berechnung des ISS eines Unfallopfers wird in $\bullet$ Tab. 1 gegeben.

Bei geeignet erscheinenden Fragestellungen wurde die statistische Signifikanz der Ergebnisse mittels $\mathrm{Chi}^{2}$-Test bestimmt.

Für die Diskussion der Ergebnisse wurden Vergleichsdaten aus der Literatur und aus „GENESIS-Online“, der Hauptdatenbank des Statistischen Bundesamtes, herangezogen.

\section{Ergebnisse}

\section{Alter und Geschlecht}

In dem untersuchten Zeitraum von 2005 bis 2016 ereigneten sich insgesamt 87 tödliche Arbeitsunfälle. Es entfielen 84 (96,6\%) Unfälle auf männliche und $3(3,4 \%)$ auf weibliche Arbeitnehmer. Der Unterschied in der Geschlechterverteilung war statistisch signifikant $(p<0,00001)$.

Die Altersstruktur reichte vom jugendlichen Alter bis in das Rentenalter. In der Altersgruppe der 46- bis 50-
Jährigen waren mit 15 (17,2\%) Fällen die meisten Unfälle zu verzeichnen, gefolgt von der Altersklasse der 51- bis 55-Jährigen mit 14 (16,1\%) Unfällen (- Abb. 1).

\section{Body-Mass-Index}

Die Mehrheit der untersuchten tödlichen Arbeitsunfälle war nach BMI normalgewichtig $(37,9 \%)$ oder präadipös $(35,6 \%)$. Insgesamt waren $23(26,4 \%)$ der tödlich Verunfallten mit einem BMI von $30 \mathrm{~kg} / \mathrm{m}^{2}$ und mehr übergewichtig.

\section{Nationalität}

In $48(55,2 \%)$ der insgesamt 87 Fälle waren die betroffenen Arbeitnehmer deutscher Nationalität. Es fanden sich 30 (34,5\%) ausländische Arbeitnehmer unter den Verunglückten. Dieser Anteil ist signifikant höher als der Anteil ausländischer sozialversicherter Arbeitnehmer im Baugewerbe Hessens, der im Jahr 2016 entsprechend den Angaben des Statistischen Bundesamts $25 \%$ betrug $(p=0,00604)$ [31]. Polnische Staatsbürger waren unter den ausländischen Arbeitern mit 10 Fällen am häufigsten betroffen. Von 9 (10,3\%) Unfallopfern konnte die Nationalität nicht ermittelt werden. 
Rechtsmedizin 2020 · 30:144-152 https://doi.org/10.1007/s00194-020-00372-6

(c) Der/die Autor(en) 2020

\section{Dieterich · E. Herrmann · M. Parzeller}

\section{Tod bei der Arbeit - eine Analyse tödlicher Arbeitsunfälle von 2005 bis 2016 im Obduktionsgut des Instituts für Rechtsmedizin in Frankfurt am Main}

\section{Zusammenfassung}

Hintergrund. Das genaue Wissen um die Umstände eines jeden tödlichen Arbeitsunfalls ist Voraussetzung für die Identifizierung von Unfallschwerpunkten und ermöglicht eine effektive Präventionsarbeit. Mit dieser rechtsmedizinischen Studie zum Arbeitsunfallgeschehen soll ein Beitrag dazu geleistet werden, die Zahl tödlicher Arbeitsunfälle in Deutschland zu senken.

Material und Methode. Zur Untersuchung kamen die tödlichen Arbeitsunfälle, die sich im Einzugsbereich des rechtsmedizinischen Instituts Frankfurt am Main in den Jahren von 2005 bis 2016 ereigneten. Ausgewertet wurden Obduktionsprotokolle sowie die dem Institut zur Verfügung gestellten staatsanwaltschaftlichen Ermittlungsakten.
Ergebnisse. Es fanden sich 87 tödliche Arbeitsunfälle in dem genannten Zwölfjahreszeitraum. Die Altersstruktur reichte vom jugendlichen Alter bis in das Rentenalter. Betroffen waren zum größten Teil männliche Arbeiter $(96,6 \%, p<0,0001)$, verhältnismäßig häufig ausländischer Nationalität (34,5\%). Die meisten Unfälle ereigneten sich in der 2. Jahreshälfte (58,6\%), an Montagen ( $26,4 \%)$, kurz vor und nach der Mittagspause. In 3 Fällen lag die Blutalkoholkonzentration über 0,5\%o. Die Baubranche (55,2\%) war der unfallträchtigste Wirtschaftszweig. Der Absturz (28,7\%) war der häufigste Unfallmechanismus und das Polytrauma $(39,1 \%)$ gemeinsam mit dem Schädel-Hirn-
Trauma (24,1\%) gemäß dem ISS die häufigste Todesursache.

Diskussion. Nach den Ergebnissen dieser Studie sollten Alter der Arbeiter sowie die Tages-, Wochen- und Jahreszeit bei der Ausführung risikoreicher Arbeiten im Baugewerbe berücksichtigt werden. Besonderes Augenmerk sollten Arbeitgeber auf die Kontrolle von Sicherheitsvorkehrungen bei Arbeiten in der Höhe sowie auf die Durchsetzung der Helmpflicht gerade auch bei ausländischen Arbeitnehmern legen.

\section{Schlüsselwörter}

Tödlicher Arbeitsunfall · Unfallanalyse . Unfallprävention · Absturz · International Severity Score

\section{Death at work-An analysis of fatal work accidents based on autopsies performed at the Institute of Legal Medicine in Frankfurt am Main from 2005 to 2016}

\section{Abstract}

Background. Precise knowledge of the circumstances of each fatal work accident is a prerequisite to identify accident hot spots and to enable effective preventive measures. This forensic medical study on work accidents was initiated to contribute to attempts to reduce the number of fatal work accidents in Germany.

Methods. Fatal work accidents that occurred between 2005 and 2016 in the catchment area of the Institute of Legal Medicine in Frankfurt am Main were investigated. Besides autopsy protocols the files of the public prosecutor's office were also evaluated.

Results. During the 12 -year period 87 fatal work accidents were documented. The age structure of the persons involved ranged from adolescence to retirement age. Male workers $(96.6 \%, p<0.0001)$, often of foreign nationality (34.5\%) represented the majority of cases. Most accidents occurred in the second half of the year (58.6\%), on Mondays $(26.4 \%)$, shortly before and after the lunch break. In three cases the blood alcohol concentration was found to be above $0.05 \%$. The highest number of accidents occurred in the building sector (55.2\%). Falling from heights (28.7\%) was the most frequent accident and polytrauma (39.1\%) together with craniocerebral trauma (24.1\%) were the most frequent causes of death according to the ISS.
Conclusion. According to the results of this study when high-risk work in the building industry is carried out, the age of workers, the time of day, week and year should be taken into consideration. Employers should pay special attention to the control of safety precautions when work is done at heights, enforcing the obligation to wear helmets, particularly when foreign workers are concerned.

\section{Keywords}

Fatal work accident - Accident analysis . Accident prevention - Fall from heights . International severity score

\section{Wirtschaftszweige}

Der Großteil der untersuchten tödlichen Arbeitsunfälle ereignete sich mit 48 (55,2\%) Fällen im Baugewerbe. Innerhalb des Baugewerbes ereigneten sich die meisten Unfälle auf Hochbauund Montagebaustellen (58,3\%). In dem Wirtschaftszweig Verkehr und Lagerei verstarben 10 (11,5\%) Arbeitnehmer nach einem Arbeitsunfall. Jeweils 9 (10,3\%) tödliche Arbeitsunfälle fanden sich im verarbeitenden Gewerbe sowie in der Land- und Forstwirtschaft. Die übrigen 11 (12,6\%) Arbeitsunfälle ereigneten sich in Wirtschaftszweigen, die unter Sonstiges zusammengefasst wurden.

\section{Unfalljahr und Unfallmonat}

In dem untersuchten Zeitraum sank die Anzahl tödlicher Arbeitsunfälle von 11 (12,6\%) im Jahr 2005 auf 5 (5,7\%) im
Jahr 2016. Das Maximum tödlicher Arbeitsunfälle wurde 2006 mit 13 (14,9\%) Unfällen erreicht. In den Jahren 2010 und 2014 lag die Anzahl tödlicher Arbeitsunfälle mit jeweils 3 Fällen (3,4\%) am niedrigsten. In den Jahren von 2011 bis 2013 blieb die Anzahl tödlicher Arbeitsunfälle mit 6 bis 7 Vorkommnissen nahezu konstant. Im Jahr 2014 sank diese Zahl um mehr als die Hälfte auf 3 tödliche Arbeitsunfälle, um dann von 4 Fällen 


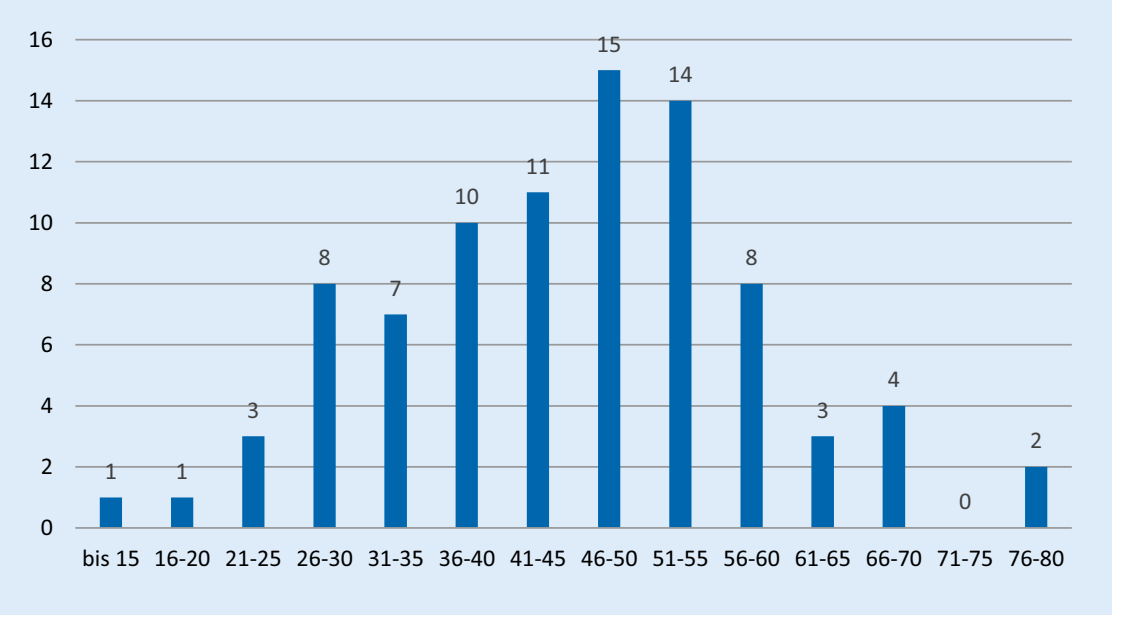

Abb. 1 A Anzahl tödlicher Arbeitsunfälle nach Lebensjahren

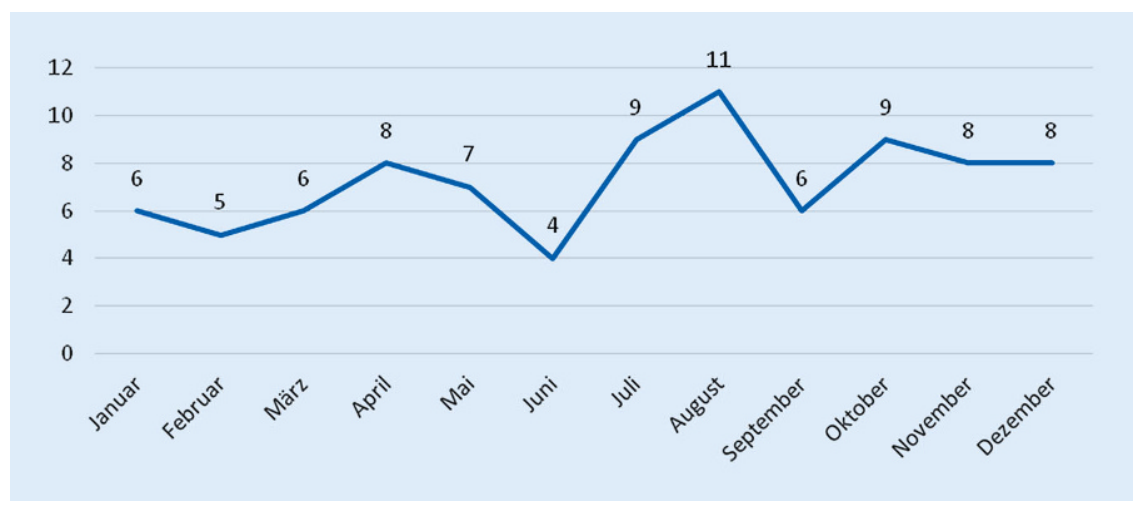

Abb. 2 A Anzahl tödlicher Arbeitsunfälle nach Unfallmonat im Jahr 2015 auf 5 Fälle im Jahr 2016 zu steigen.

Der unfallträchtigste Monat mit insgesamt $11(12,6 \%)$ tödlichen Unfällen war der Sommermonat August. Im Frühlingsmonat Juni waren mit 4 (4,6\%) Fällen die wenigsten tödlichen Arbeitsunfälle zu verzeichnen. Ein statistisch signifikanter Unterschied zwischen den Monaten konnte nicht nachgewiesen werden $(p=0,8846)$. In der zweiten Jahreshälfte wurden mit 51 (58,6\%) Fällen im Vergleich zu der ersten Jahreshälfte mit 36 $(41,4 \%)$ Fällen mehr Unfälle beobachtet (•Abb. 2).

\section{Unfalltag und Unfalluhrzeit}

Die meisten Unfälle ereigneten sich mit $23(26,4 \%)$ Fällen an Montagen. Im weiteren Verlauf der Woche stieg die Anzahl tödlicher Arbeitsunfälle annähernd proportional von 13 (14,9\%) an

\section{Unfallmechanismus}

Häufigste Unfallmechanismen waren der Absturz mit 25 (28,7\%) und das Getroffenwerden von Gegenständen mit $24(27,6 \%)$ Fällen. Es folgten schwere Quetschtraumata mit $15(17,2 \%)$ und Überrolltraumata mit 6 (6,9\%) Unfällen. Verbrennungen und Vergiftung mit Todesfolge sowie das Ertrinken und der Stromtod spielten mit jeweils einem bis 3 Fällen eine untergeordnete Rolle. Bei 9 (10,3\%) tödlichen Arbeitsunfällen ließ sich der Unfallmechanismus keiner der Kategorien zuordnen. Es handelte sich bei der großen Mehrzahl der untersuchten Arbeitsunfälle um mechanische Traumata.

\section{Absturz}

Die insgesamt 25 tödlichen Absturzunfälle ereigneten sich von Dächern (32\%), Gerüsten (32\%) und Leitern (24\%). Unter Sonstiges (12\%) wurde der Absturz in einen Fahrstuhlschacht sowie der Sturz von einem Telefonmast und von einer Maschine zusammengefasst.

Mit 20 Abstürzen entfielen $80 \%$ aller Absturzunfälle auf das Baugewerbe. Der Absturz war mit 20 (41,7\%) Fällen der häufigste Unfallmechanismus im Baugewerbe.

Die Absturzhöhe lag zwischen knapp unter 2 und $25 \mathrm{~m}$. Von der Absturzhöhe zwischen 3,0 und 6,99 m ereigneten sich mit 14 (56\%) Fällen über die Hälfte der tödlichen Abstürze (• Abb. 5).

\section{International Severity Score}

tödlichen Arbeitsunfälle waren mit insgesamt 18 (20,7\%) Fällen am erweiterten Wochenende von Freitag bis Sonntag zu verzeichnen. Für die Anzahl der Unfälle von Montag bis Donnerstag und der Arbeitsunfälle am erweiterten Wochenende konnte ein signifikanter Unterschied gezeigt werden ( $p=0,000029)$.

Bei der Auswertung des Unfallzeitpunkts zeigten sich 2 Peaks, zum einen vormittags zwischen 10:00 und 11:59 Uhr, zum anderen nachmittags zwischen 14:00 und 15:59 Uhr mit jeweils 19 $(21,8 \%)$ Unfällen. Zwischen diesen Peaks ereigneten sich von 12:00-13:59 Uhr lediglich 10 (11,5\%) Unfälle (• Abb.4).

Die beiden tiefsten Werte für den ISS wurden mit 2 und 4 Punkten nach Ertrinken resp. Verschüttung erreicht. Da in dieser Studie jedes nach ISS bewertete Verletzungsbild im Rahmen eines tödlichen Arbeitsunfalls entstanden ist, belegen die ermittelten ISS-Scores meist mittlere bis hohe Punktzahlen (• Tab. 2). Das Maximum von 75 Punkten wurde mit $9(11,1 \%)$ Fällen am häufigsten belegt. In $6(6,9 \%)$ Fällen war aufgrund einer längeren Überlebenszeit keine Codierung der Verletzungen nach der AIS möglich. 


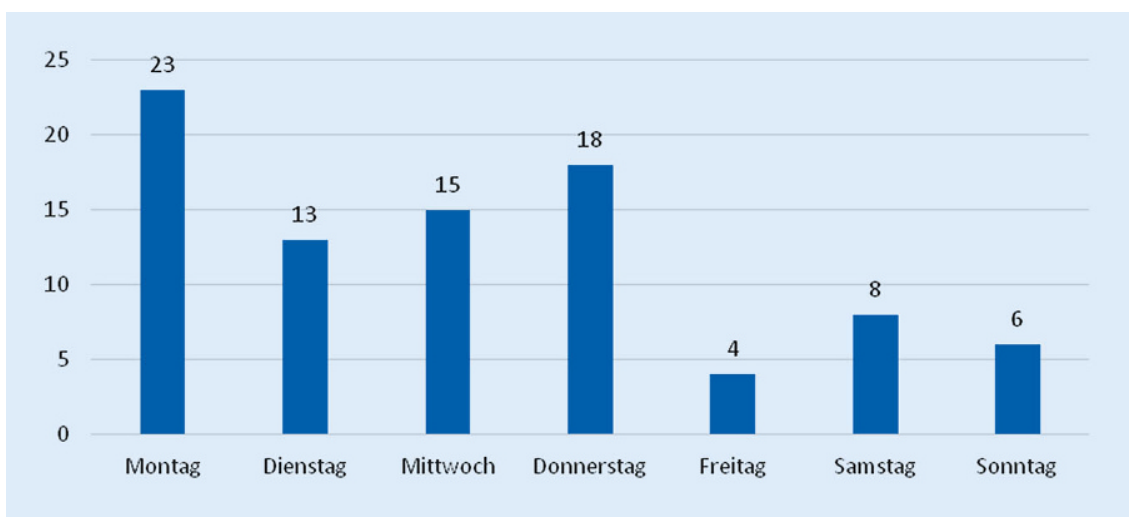

Abb. $3 \Delta$ Anzahl tödlicher Arbeitsunfälle nach Wochentag

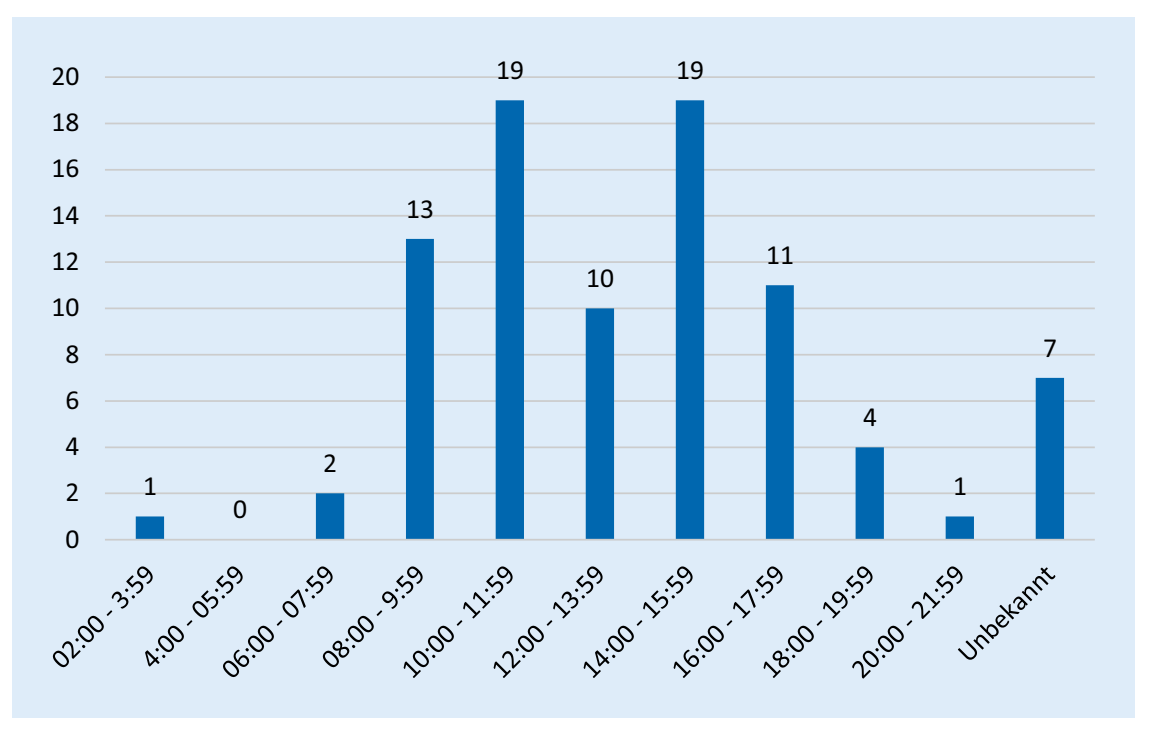

Abb. 4 \ Anzahl tödlicher Arbeitsunfälle nach Unfalluhrzeit

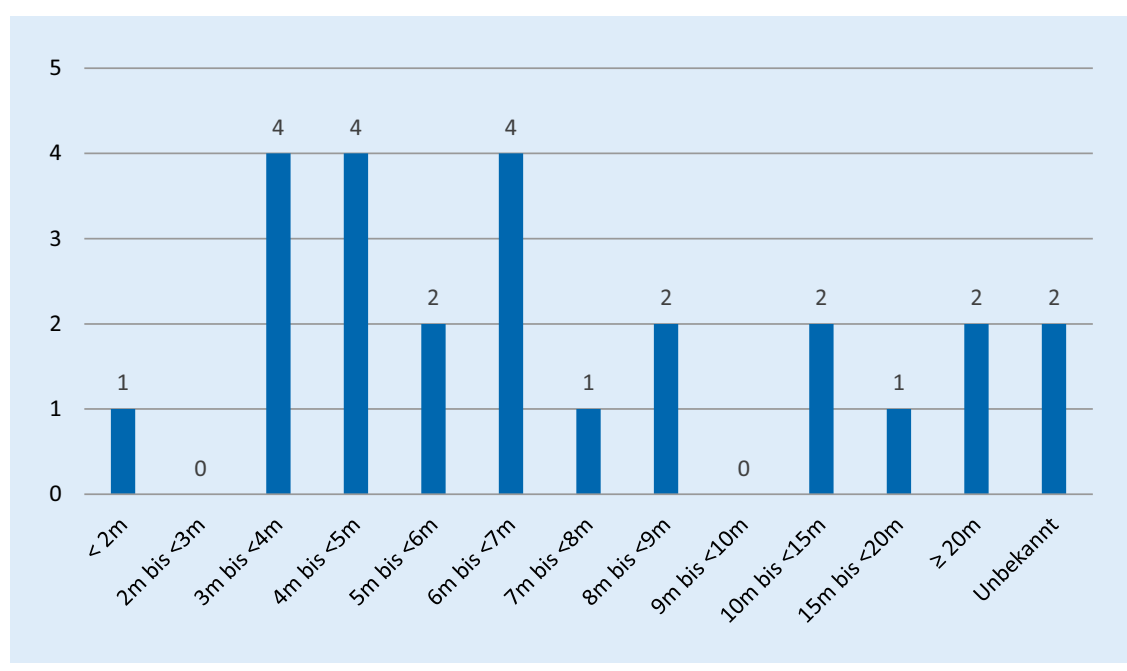

Abb. 5 \ Anzahl tödlicher Arbeitsunfälle nach Absturzhöhe in Metern

\section{Blutalkoholkonzentration und Drogen}

Die gaschromatographische Messung der BAK wurde bei 67 (77,0\%) Unfallopfern durchgeführt. Bei den übrigen 20 $(23,0 \%)$ Verunfallten erfolgte meist aufgrund einer längeren Überlebenszeit keine Bestimmung der BAK.

Bei 26 (38,8\%) der auf Alkohol im Blut untersuchten Todesopfer wurde eine BAK von $0,0 \%$ festgestellt. Weitere 31 (46,3\%) der toxikologisch untersuchten Unfallopfer wiesen eine BAK von $0-0,1 \%$ auf. Da eine geringe Menge von Alkohol im Blut aufgrund postmortaler Fäulnisprozesse entstehen kann [19], wurden diese als nichtrelevante Alkoholbeeinträchtigung gewertet.

Mit Werten von $0,1-0,5 \%$ standen $8(11,9 \%)$ Unfallopfer unter relativem Alkoholeinfluss. In 2 (3,0\%) Fällen bestand mit 0,51\%o und dem Höchstwert von 2,07\%o eine relevante Alkoholbeeinträchtigung. Beide Arbeitsunfälle ereigneten sich an einem Donnerstag.

In $3(3,4 \%)$ Fällen konnte im Rahmen der toxikologischen Untersuchung die Aufnahme von Cannabisprodukten festgestellt werden, wobei aber keine rauschwirksamen Bestandteile mehr nachweisbar waren.

\section{Todesursache}

Die häufigste Todesursache war das Polytrauma mit $34(39,1 \%)$ Fällen. Das todesursächliche Verletzungsbild wurde retrospektiv dann als Polytrauma gewertet, wenn nach ISS mindestens 2 Körperregionen betroffen waren und sich die jeweiligen Verletzungen mit nicht mehr als einem Punkt in ihrem Schweregrad voneinander unterschieden.

Die zweithäufigste Todesursache war das Schädel-Hirn-Trauma mit 21 $(24,1 \%)$ Fällen. Von den 87 Verunfallten verstarben weitere $11(12,6 \%)$ an einer Kompression des Thorax und 5 (5,7\%) durch ein Trauma der Abdominalregion. Verbrennungen waren in $3(3,4 \%)$ Fällen todesursächlich. Vergiftung, Ertrinken und Stromtod führten in jeweils $2(2,3 \%)$ Fällen zum Tod eines Unfallopfers.

Unter Sonstiges fällt eine Verletzung der Beinhauptschlagader mittels Hoch- 
Tab. 2 Verteilung der Verletzungsschwere nach dem International Severity Score (ISS)

\begin{tabular}{|l|l|}
\hline ISS-Wert & Anzahl der Arbeitsunfälle \\
\hline $1-15$ & 4 \\
$16-30$ & 20 \\
\hline $31-45$ & 26 \\
$46-60$ & 19 \\
$61-75$ & 18 \\
\hline
\end{tabular}

druckstrahler und nachfolgendem Verbluten nach außen.

An Folgezuständen verstarben 6 (6,9\%) der Verunfallten. Hierunter sind 2 Multiorganversagen nach Sepsis, 2 Herzversagen und 2 Lungenembolien zusammengefasst.

\section{Diskussion}

Die eigenen Ergebnisse wurden den Studien von Gawehn [12] und Kiehl [17], die am selben Institut vergleichbare Untersuchungen früherer Sektionsjahrgänge durchgeführt haben, gegenübergestellt.

In der Analyse der obduzierten tödlichen Arbeitsunfälle der Jahre 1979-1988 von Kiehl war die Altersgruppe der 36bis 40-Jährigen am häufigsten betroffen. Für den von Gawehn [12] untersuchten Zeitraum von 1991 bis 2004 lag das Maximum der Altersverteilung ein Jahrzehnt darüberbeiden 46- bis50-Jährigen. Diese Altersgruppe war auch in der vorliegenden Studie am häufigsten betroffen.

Vergleicht man die Altersverteilung der tödlich Verunglückten dieser Studie mit der Altersverteilung der Erwerbstätigen in Deutschland näherungsweise für das Jahr 2016 zeigt sich, dass der Anteil der Verunglückten im Verhältnis zu dem Anteil Erwerbstätiger in den Altersklassen von 26 bis 60 Jahren relativ ausgewogen ist. In den Altersklassen von 61 bis 70 Jahre ist der Anteil der tödlich Verunfallten $(8,0 \%)$ im Verhältnis zu dem Anteil der in diesen Altersklassen erwerbstätigen Personen hingegen etwa doppelt so hoch [31]. Bei dem Vergleich der Altersstruktur der Unfallopfer dieser Studie und der Altersstruktur der Erwerbstätigen in Hessen zeigt sich ein ausgewogenes Verhältnis der Altersklassen von 18 bis 64 Jahren. Der Anteil der bei der Arbeit tödlich Verunglückten von 65 und mehr Jahren ist wiederum etwa doppelt so hoch wie der Anteil gleichaltriger Erwerbstätiger in Hessen [31].

Diese relative Zunahme der tödlichen Arbeitsunfälle ab 61 Jahren legt einen Zusammenhang tödlicher Arbeitsunfälle mit der Abnahme der körperlichen Leistungsfähigkeit im Alter nahe [27]. Die mit dem demografischen Wandel in Deutschland einhergehende Veränderung der Altersstruktur der Bevölkerung [31] könnte zukünftig der Prävention tödlicher Arbeitsunfälle entgegenstehen. Arbeitgeber sollten dazu angehalten werden, ältere Arbeitnehmer verstärkt in risikoarmen Arbeitsbereichen einzusetzen.

Der niedrige Anteil von Frauen (3,4\%) am tödlichen Arbeitsunfallgeschehen für den untersuchten Zeitraum von 2005 bis 2016 lässt sich zum einen auf die niedrige Frauenquote in dem unfallträchtigen Baugewerbe zurückführen. Laut dem Statistischen Bundesamt lag 2016 der Anteil weiblicher Erwerbstätiger im Baugewerbe bei 15,3\% [31]. Zum anderen ist denkbar, dass weibliche Arbeitnehmer in risikoärmeren und physisch weniger belastenden Arbeitsbereichen eingesetzt werden.

Auch bei Gawehn [12] und Kiehl [17] sowie in weiteren rechtsmedizinischen Untersuchungen anderer Institute [7, 18, $24,28]$ fanden sich niedrige Anteile weiblicher Arbeitnehmer an dem tödlichen Arbeitsunfallgeschehen mit Werten zwischen 0 und $6,2 \%$.

Neben dem Alter über 60 Jahren sowie dem männlichen Geschlecht ist nach dieser Untersuchung auch die Zugehörigkeit zu einer ausländischen Nationalität ein Risikofaktor, einen tödlichen Arbeitsunfall zu erleiden. Der Anteil von 34,4\% ausländischer Unfallopfer dieser Studie deckt sich nicht mit dem Anteil ausländischer sozialversicherungspflichtig Beschäftigter in Hessen. Dieser betrug für das Jahr 2016 lediglich 13,6\% [31]. Auch für das Baugewerbe findet sich in Hessen im Jahr 2016 lediglich ein Anteil von 25,0 \% ausländischer Beschäftigter [31].

Im zeitlichen Verlauf hat der Anteil ausländischer Unfallopfer unter den in der Rechtsmedizin Frankfurt am Main obduzierten tödlichen Arbeitsunfällen zuletzt zugenommen. In dem Zeitraum von 1991 bis 2004 betrug der Anteil ausländischer Arbeitnehmer unter den tödlichen Arbeitsunfällen 23\% [12].

Ein möglicher Grund für den hohen Anteil ausländischer Arbeitnehmer am tödlichen Arbeitsunfallgeschehen sind fehlende Sprachkenntnisse. Förderung von Sprachunterricht sowie das Vermitteln von Sicherheitsbestimmungen mittels Piktogrammen und audiovisuellem Material können dazu beitragen, ausländischen Arbeitnehmern individuelle Sicherheitsrisiken bei der Arbeit besser verständlich zu machen [16].

Im Vergleich der männlichen Unfallopfer dieser Studie zu der männlichen Gesamtbevölkerung Deutschlands zeigen sich hinsichtlich der BMI-Werte geringe Unterschiede. In dieser Studie waren $27,1 \%$ der männlichen Unfallopfer mit einem BMI von $30 \mathrm{~kg} / \mathrm{m}^{2}$ oder mehr übergewichtig. Im Jahr 2017 lag laut Mikrozensus des Statistischen Bun-
Hier steht eine Anzeige. Springer 
desamts der Anteil übergewichtiger, männlicher Bundesbürger ab 18 Jahren bei $18,1 \%$ [31].

Die in dieser Studie unfallträchtigsten Monate August (12,6\%), Juli (10,3\%) und September $(10,3 \%)$ liegen in der auftragsreichen Sommerarbeitszeit des Baugewerbes von April bis November. Die Häufung von tödlichen Arbeitsunfällen zur Sommerzeit deckt sich mit den Ergebnissen in der Literatur [2, 12, 15, 28, 29]. Nach dem Tarifrecht des unfallträchtigen Baugewerbes werden in der Sommerarbeitszeit 41 h und in der Winterarbeitszeit lediglich $38 \mathrm{~h}$ gearbeitet. In der Sommerzeit ist die Expositionszeit der Arbeiter gegenüber dem Risiko, einen Arbeitsunfall zu erleiden, entsprechend hoch. $\mathrm{Zu}$ dem beeinträchtigen die erhöhten Temperaturen zur Sommerzeit die Arbeiter in ihrer Aufmerksamkeit und können zu einer frühzeitigen Ermüdung führen [1]. Das Einhalten regelmäßiger Pausenzeiten, die Anpassung der Bekleidung an das Umgebungsklima [20] und die Ausführung risikoreicher, körperlich anstrengender Arbeiten zu klimatisch günstigen Jahres- und Tageszeiten helfen, der Erschöpfung der Arbeiter entgegenzuwirken. Diese Präventionsmaßnahmen könnten vor dem Hintergrund des Klimawandels zukünftig noch an Bedeutung zunehmen.

Im Zeitraum von 2005 bis 2016 ereigneten sich im Einzugsgebiet der Rechtsmedizin Frankfurt am Main die meisten tödlichen Arbeitsunfälle montags. Der Montag wird auch in der Literatur als häufigster Arbeitsunfalltag ermittelt [6, 7, 10, 12, 21, 22, 29]. Entgegen der von Gawehn [12] beschriebenen Verstärkung der Montagshäufung durch vermehrte Krankmeldungen, ist der „blaue Montag"laut Erhebungen mehrerer deutscher Krankenkassen ein Mythos. Die Barmer Ersatzkasse stellte für das Jahr 2017 eine Häufung der Krankmeldungen von Mittwoch bis Freitag mit einem Anteil von 15,4-15,7\% [11] und die Allgemeine Ortskrankenkasse (AOK) für dasselbe Jahr eine Häufung der Krankmeldungen an Dienstagen mit 19,2\% [23] fest. Für den Montag fanden sich lediglich Anteile von 13,9\% bzw. 12,7\% respektive. Es konnten keine Bezugsdaten ermittelt werden, ob an Montagen, der geringeren Anzahl an Krankmeldungen entsprechend, tatsächlich die meisten Arbeitsstunden verrichtet wurden, wodurch eine Häufung tödlicher Arbeitsunfälle an diesem Wochentag erklärt werden könnte. Ein denkbarer Grund für die Montagshäufung sind mögliche physische und psychische Anpassungsschwierigkeiten bei der Umstellung von dem freien Wochenende auf die arbeitsspezifischen Risiken zum Wochenbeginn.

Zwischen den ermittelten Hauptunfallzeiten von 10:00-11:59 Uhr sowie von 14:00-15:59 Uhr halbiert sich die Anzahl tödlicher Arbeitsunfälle beinahe. In diese Zeit fällt üblicherweise die Mittagspause. Ein ähnlicher Kurvenverlauf mit 2 von der Mittagszeit getrennten Peaks findet sich auch in der Literatur [9, 15, 17, 18, 22, 26, 29]. Die Untersuchung von Schieche [28] zeigte einen gegenläufigen Trend. Hier lag das Maximum der zeitlichen Häufigkeitsverteilung tödlicher Arbeitsunfälle in der Mittagspause zwischen 12:00 und 13:59 Uhr. Auch Bratzke und Hamoser [7] sowie Gawehn [12] konnten keinen Abfall der Unfallhäufigkeit während der Mittagspause feststellen. Eine Begründung findet sich hierfür, abgesehen von evtl. flexiblen Mittagspausenzeiten im Baugewerbe, nicht.

Von den auf BAK untersuchten Unfallopfern ist der Anteil relativer (11,9\%) und relevanter (3,0\%) Alkoholbeeinträchtigung im Vergleich $\mathrm{zu}$ früheren Untersuchungen gering. Bei Gawehn [12] war im untersuchten Zeitraum von 1991 bis 2004 der Anteil relevanter Alkoholbeeinträchtigung mit 2,9\% ebenfalls niedrig. Bei der Erhebung am selben Institut von Kiehl [17] fand sich zwischen 1979 und 1988 ein Anteil von 19,1\% mit BAK über 0,5\%o. An anderen rechtsmedizinischen Instituten fand Schieche [28] für dem Zeitraum von 1990 bis 1995 in Berlin einen Anteil relevanter Alkoholbeeinträchtigung von $17,8 \%$, Bartsch [4] von 1971 bis 1983 in Hamburg von $26,8 \%$ und Naeve, Brinkmann et al. [25] von 1962 bis 1970 in Hamburg von 39\%. In der Zusammenschau der genannten Untersuchungen hat sich das Problem der tödlichen Arbeitsunfälle unter Alkoholeinfluss im Verlauf der vergangenen Jahrzehnte gebessert.
Anhand der Analyse des Unfallhergangs lassen sich weitere Erkenntnisse für die Prävention tödlicher Arbeitsunfälle gewinnen. In dem untersuchten Obduktionsgut war der Absturz mit 25 (28,7\%) Fällen der häufigste Unfallmechanismus. Auch die Vergleichsarbeiten des Instituts für Rechtsmedizin Frankfurt am Main von Gawehn (41,9\% Abstürze) [12] und Kiehl (32,5\% Abstürze) [17] identifizierten den Absturz als Hauptunfallursache. Tatsächlich finden sich im bundesweiten Vergleich fast ausschließlich Studien, die den Absturz als häufigste Unfallursache bei der Arbeit angeben $[4,7,8,18,28$, 30].

Die meisten Absturzunfälle ereigneten sich von Dächern, Gerüsten und Leitern und von Höhen zwischen 3,0 und $6,99 \mathrm{~m}$. Im Baugewerbe war der Absturz mit 20 (41,7\%) Fällen besonders häufig. Der $\$ 12$ der DGUV-Vorschrift 38/BGV C 22 der Deutschen Gesetzlichen Unfallversicherung sieht für alle Arbeitsplätze ab $2 \mathrm{~m}$ Absturzhöhe eine Absturzsicherung vor. Ausnahmen für die verpflichtende Absturzsicherung bilden Arbeitsplätze auf Dächern bis 2,99 m Absturzhöhe sowie beim Mauern über die Hand und beim Arbeiten an Fenstern bei einer Absturzhöhe bis zu 4,99 m. Diese Maßnahmen gewährleisten, auch vor dem Hintergrund nur eines ermittelten Unfalls mit einer Absturzhöhe unter $3 \mathrm{~m}$, in der Theorie einen sicheren Schutz vor tödlichen Absturzunfällen. Möglicherweise ist der Wille zur praktischen Umsetzung solcher Präventionsmaßnahmen nicht immer gegeben, v. a. hinsichtlich der Praktikabilität bei Arbeiten kurzer Dauer. Durch engmaschige Kontrollen der Sicherheitsvorkehrungen bei Arbeiten in der Höhe und durch intensive Schulungen zu dem arbeitsspezifischen Risiko des tödlichen Absturzes bei Arbeiten auf Gerüsten, Leitern oder Dächern könnten tödliche Arbeitsunfälle durch Abstürze zukünftig reduziert werden.

Bei der Auswertung der Obduktionsprotokolle der 87 untersuchten tödlichen Arbeitsunfälle war das Polytrauma mit 34 Fällen $(39,1 \%)$ die häufigste Todesursache. Innerhalb der Gruppe polytraumatisierter Unfallopfer war der Kopf mit 20 (58,8\%) Fällen die 
am häufigsten verletzte Region unter den 3 jeweils am stärksten betroffenen Körperregionen. Zusammen mit den $21(24,1 \%)$ todesursächlichen SchädelHirn-Traumata ergeben sich $41(47,1 \%)$ tödliche Arbeitsunfälle, bei denen das Schädel-Hirn-Trauma eigenständig oder zumindest maßgeblich todesursächlich war. Demzufolge liegt es nahe, als allgemeine und einfache Schutzmaßnahme vor Schädel-Hirn-Verletzungen eine verstärkte Durchsetzung der Helmpflicht im Rahmen der Gefährdungsbeurteilung von Baustellen abzuleiten, welche laut $\$ \$ 4$ und 5 ArbSchG (Arbeitsschutzgesetz) dem Arbeitgeber obliegt. Mittels unangemeldeter Kontrollen sollten Arbeitnehmer durch den Arbeitgeber an ihre Verpflichtung nach $\$ 15$ Absatz 2 ArbSchG erinnert werden, die persönliche Schutzausrüstung bestimmungsgemäß zu verwenden.

\section{Fazit für die Praxis}

- Arbeitgeber sollten dazu angehalten werden, ältere Arbeiter in risikoarmen Arbeitsbereichen einzusetzen.

- Durch die Förderung von Sprachunterricht sollten ausländischen Arbeitnehmern die individuellen Sicherheitsrisiken bei der Arbeit besser verständlich gemacht werden.

- Für eine wirkungsvolle Präventionsarbeit sollten Arbeiter für die Häufung tödlicher Arbeitsunfälle in den Sommermonaten, an Montagen sowie kurz vor und direkt nach der Mittagspause sensibilisiert werden.

- Durch engmaschige Kontrollen der Sicherheitsvorkehrungen bei Arbeiten in der Höhe und durch intensive Schulungen zu dem arbeitsspezifischen Risiko des tödlichen Absturzes bei Arbeiten auf Gerüsten, Leitern oder Dächern könnten tödliche Arbeitsunfälle durch Abstürze zukünftig reduziert werden.

- Erfahrungswerte beim Tragen von Schutzbekleidungen (z. B. Helmpflicht bei Motorradfahrern) sprechen dafür, dass das verstärkte Durchsetzen einer Helmpflicht durch den Arbeitgeber auf Baustellen im Hinblick auf die hohe Anzahl tödlicher Schädel-Hirn-Traumata zum
Erreichen der „vision zero" beitragen könnte.

\section{Korrespondenzadresse}

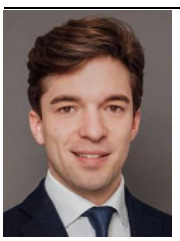

\section{Dieterich}

Institut für Rechtsmedizin, Universitätsklinikum, GoetheUniversität Frankfurt a. M. Frankfurt a. M., Deutschland constantin.dieterich@ outlook.de

Danksagung. Die Autoren bedanken sich bei Herrn Prof. Dr. Mebs für die Übersetzungshilfe.

Funding. Open Access funding provided by Projekt DEAL.

\section{Einhaltung ethischer Richtlinien}

Interessenkonflikt. C. Dieterich, E. Herrmann und M. Parzeller geben an, dass kein Interessenkonflikt besteht.

Alle beschriebenen Untersuchungen am Menschen oder an menschlichem Gewebe wurden mit Zustim mung der zuständigen Ethik-Kommission, im Einklang mit nationalem Recht sowie gemäß der Deklaration von Helsinki von 1975 (in der aktuellen, überarbeiteten Fassung) durchgeführt. Die Untersuchungen erfolgten unter Einhaltung der Vorgaben der Zentralen Ethikkommission der Bundesärztekammer.

Open Access. Dieser Artikel wird unter der Creative Commons Namensnennung 4.0 International Lizenz veröffentlicht, welche die Nutzung, Vervielfältigung, Bearbeitung, Verbreitung und Wiedergabe in jeglichem Medium und Format erlaubt, sofern Sie den/die ursprünglichen Autor(en) und die Quelle ordnungsgemäß nennen, einen Link zur Creative Commons Lizenz beifügen und angeben, ob Änderungen vorgenommen wurden.

Die in diesem Artikel enthaltenen Bilder und sonstiges Drittmaterial unterliegen ebenfalls der genannten Creative Commons Lizenz, sofern sich aus der Abbildungslegende nichts anderes ergibt. Sofern das betreffende Material nicht unter der genannten Creative Commons Lizenz steht und die betreffende Handlung nicht nach gesetzlichen Vorschriften erlaubt ist, ist für die oben aufgeführten Weiterverwendungen des Materials die Einwilligung des jeweiligen Rechteinhabers einzuholen.

Weitere Details zur Lizenz entnehmen Sie bitte der Lizenzinformation auf http://creativecommons.org/ licenses/by/4.0/deed.de.

\section{Literatur}

1. Adam-Poupart A, Smargiassi A, Busque M-A et al (2015) Effect of summer outdoor temperatures on work-related injuries in Quebec (Canada). Occup Environ Med 72(5):338-345
2. Amiri M, Ardeshir A, Fazel Zarandi MH (2014) Risk-based analysis of construction accidents in Iran during 2007-2011-meta analyze study. Iran J Public Health 43(4):507-522

3. Baker SP, O'Neill B, Haddon W et al (1974) The injury severity score: a method for describing patients with multiple injuries and evaluating emergency care. JTrauma 14(3):187-196

4. Bartsch N (1987) Analyse der tödlichen Betriebsunfälle in Hamburg der Jahre 1971 bis 1983 unter besonderer Berücksichtigung des Alkoholeinflusses, Diss. Hamburg

5. Betz P, Eisenmenger W (1992) Vorsätzliche Tötung mit Vortäuschen eines Arbeitsunfalles. Arch Kriminol 190:151-162

6. Böthig R, Lignitz E (1991) Tödliche Unfälle in Berlin (Ost) 1985-1987. In: Schütz H, Kaatsch H-J, Thomsen H (Hrsg) Medizinrecht - Psychopathologie - Rechtsmedizin. Springer, Berlin, Heidelberg, S349-361

7. Bratzke H, Hammoser C (1983) Tödliche Arbeitsunfälle in Berlin (West) aus forensischer Sicht. Beitr Gerichtl Med 41:263-270

8. Bundesanstalt für Arbeitsschutz und Arbeitsmedizin (2017) Tödliche Arbeitsunfälle. baua, Dortmund

9. Camino López MA, Fontaneda I, González Alcántara OJ et al (2011) The special severity of occupational accidents in the afternoon: "the lunch effect". Accid Anal Prev 43(3):1104-1116

10. Campolieti M, HyattDE (2006) Further evidence on the "monday effect" in workers' compensation. Ind Labor Relat Rev 59(3):438-450

11. Grobe TG, Steinmann S, Gerr J (2018) Gesundheitsreport 2018. Müller Verlagsservice e.K., Siegburg

12. Gawehn S (2007) Tödliche Arbeitsunfälle im Sektionsgut der Rechtsmedizin Frankfurt am Main 1991-2004, Diss. Frankfurta. M.

13. Haasper C, Junge M, Ernstberger A et al (2010) Die Abbreviated Injury Scale (AIS). Potenzial und Probleme bei der Anwendung. Unfallchirurg 113(5):366-372

14. Hämäläinen P, Nenonen N, Saarela K, Takala J et al (2014) Global estimates of occupational accidents and work-related illnesses 2014, made for the ILO report at XX world congress, Frankfurt. https://doi. org/10.13140/2.1.2864.0647

15. Huang X, Hinze J (2003) Analysis of construction worker fall accidents. J Constr Eng Manag 129(3):262-27

16. De Jesus-Rivas M, Conlon HA, Burns C (2016) The impact of language and culture diversity in occupational safety. Workplace Health Saf 64(1):24-27

17. Kiehl C (1990) Eine Analyse von tödlichen Arbeitsunfällen aus den Jahren 1979-1988 unter Berücksichtigung der Ursachen und der rechtlichen Folgen, Diss. Frankfurt a. $M$.

18. Klima M (1987) Tödliche Arbeitsunfälle in den Jahren 1974, 1975, 1976 und 1982 sowie Auswertung des Obduktionsgutes der Jahre 1974 und 1975 des Instituts für Rechtsmedizin de Ludwig-Maximilians-Universität zu München, Diss. München

19. Kugelberg FC, Jones AW (2007) Interpreting results of ethanol analysis in postmortem specimens: a review of the literature. Forensic Sci Int 165(1):10-29

20. Leyk D, Hoitz J, Becker C et al (2019) Health risks and interventions in exertional heat stress. Dtsch Arztebl Int 116(31-32):537-544 
21. López Arquillos A, Rubio Romero JC, Gibb A (2012) Analysis of construction accidents in Spain, 2003-2008. J Safety Res 43(5-6):381-388

22. Mason K (1979) Accident patterns by time-of-day and day-of-week of injury occurrence. J Occup Accid 2(2):159-176

23. Meyer M, Wenzel J, Schenkel A (2018) Krankheitsbedingte Fehlzeiten in der deutschen Wirtschaft im Jahr 2017. In: Badura B, Ducki A, Schröder H, Klose J, Meyer M (Hrsg.) Fehlzeiten-Report 2018. Springer Berlin Heidelberg, Berlin, Heidelberg, S331-536

24. Moschkau N, Kunz S, Fieseler S et al (2011) Sektionsdaten des Instituts für Rechtsmedizin der Universität München aus dem Jahr 2003. Rechtsmedizin 21(6):541-548

25. Naeve W, Brinkmann B, Janssen W (1973) Alkohol und Betriebsunfall. Gerichtsmedizinischer Beitrag zur sozialmedizinischen Bedeutung alkoholbedingter Betriebsunfälle. Beitr Gerichtl Med 30:317-323

26. Pantke F (1970) Über tödliche Arbeitsunfälle in West-Berlin von 1960 bis 1965 unter der Berücksichtigung der Alkoholbeeinflussung, Diss. Berlin

27. Rinkenauer G (2008) Motorische Leistungsfähigkeit im Alter. In: Schlag B (Hrsg) Leistungsfähigkeit und Mobilität im Alter. TÜV Media, Köln, S143-180

28. Schieche C (2000) Tödliche Arbeitsunfälle in Berlin der Jahre 1990 bis 1995 - Eine rechtsmedizinische Analyse, Diss. Berlin

29. Shao B, Hu Z, Liu Q et al (2019) Fatal accident patterns of building construction activities in China. Saf Sci 111:253-263

30. Siefer A, Hermanns D (2004) Tödliche Arbeitsunfälle 2001-2002. Wirtschaftsverl. NW, Dortmund

31. Statistisches Bundesamt (Destatis) (2020) GenesisOnline

32. World Health Organization (2000) Obesity-preventing and managing the global epidemic. WHO, Genf

\section{Das Leben genommen - Suizid im Kindes- und Jugendalter}

Suizide sind nach Unfällen die zweithäufigste Todesursache bei Kindern und Jugendlichen in Deutschland, aber die absoluten Zahlen haben sich in den letzten Jahren kaum verändert. Die World Federation for Mental Health verweist auf die unterschätze Bedeutung von suizidalem Verhalten unter Kinder und Jugendlichen.

Suizidversuche und vollendete Suizide berühren nicht nur die Betroffenen, sondern auch ihre Familien und ihr soziales Umfeld einschneidend. Die Ursachen für Suizide von Kindern und Jugendlichen sind vielfältig. Medienberichte zum Thema können in Verbindung mit Hinweisen auf Hilfsangebote eine suizidpräventive Wirkung bei Kindern und Jugendlichen entfalten (sog. „Papageno"-Effekt), innen aber auch schaden (sog. "Werther-Effekt"). Daher ist ein verantwortungsvoller Umgang mit dem Thema in der medialen Öffentlichkeit unerlässlich.

\section{"Werther-Effekt"}

Dass dies nicht immer gegeben ist, zeigt die US-amerikanische Serie „Tote Mädchen lügen nicht" (im englischsprachigen Original „13 Reasons Why"). Sie beschreibt detailliert die belastenden Umstände, denen die jugendliche Protagonistin ausgesetzt ist, bevor sie sich schließlich selbst das Leben nimmt. Aus diesem Grund hatte die DGKJP im Jahr 2017 zusammen mit der DGPPN die Serie problematisiert und Empfehlungen zum Umgang formuliert (www.dgkjp.de/stellungnahmen-positionspapiere/stellungnahmen-2017/453-gemeinsame-stellungnahme-tv-serie-tote-maedchen-luegen-nicht-2). Kürzlich erbrachte eine wissenschaftliche Studie den Beleg, dass infolge der Ausstrahlung der Serie sich mehr 10- bis 17-Jährige in den USA das Leben genommen haben. Erst dann hat Netflix auf die anhaltende Kritik reagiert und die Darstellung des Suizids gekürzt und entschärft.

\section{Fachpersonal stark gefordert}

Suizidales Verhalten von Kindern und Jugendlichen ist auch für Menschen, die beruflich mit ihrer Versorgung betraut sind, eine stark fordernde Situation. Stress, Schuldgefühle und Hilflosigkeit sind typische Symptome bei therapeutisch, pflegerisch und pädagogisch tätigem Fachpersonal. Eine deutliche Mehrheit derer, die mit jungen Menschen mit psychischen Störungen arbeiten, hatte bereits mit Suizidversuchen von Patient*innen zu tun (94\%) - dies zeigt eine aktuelle Umfrage der DGKJP zum Thema "Suizid bei Kindern und Jugendlichen", an der 270 Mitglieder teilgenommen haben. Ein Viertel der Befragten (26\%) berichtet dabei, mit mehr als 10 Suizidversuchen in den letzten 12 Monaten konfrontiert gewesen zu sein. Vollendete Suizide gehören ebenfalls zum Erfahrungsspektrum der Befragten (56\%). Zur fachlichen Unterstützung der Personen, die mit psychisch erkrankten jungen Menschen arbeiten, hat die DGKJP in Zusammenarbeit mit anderen Fachgesellschaften die Leitlinie „Suizidalität im Kindes- und Jugendalter" erstellt (AWMF-Register-Nr. 028/031).

Quelle: Deutsche Gesellschaft für Kinder- und Jugendpsychiatrie, Psychosomatik und Psychotherapie e.V. (DGKJP) 